\title{
Influencia de los estilos parentales de crianza en el pensamiento divergente y sentimiento divergente
}

\author{
Influence of Parental Styles on the Divergent Thinking \\ and Divergent Feeling \\ Carmen Lucía Neira Belón \\ Universidad Católica San Pablo, Arequipa, Perú \\ (iD) https://orcid.org/oooo-0oo2-3950-3348 \\ Correspondencia: lucia.neira.belonı@gmail.com
}

\begin{abstract}
Resumen
El presente artículo expone el análisis de la asociación de los estilos parentales de crianza y el desarrollo del pensamiento divergente y sentimiento divergente en adolescentes. Dado que la familia es importante para el desarrollo de los niños y estudios previos sugieren que el estilo parental puede influenciar en la creatividad, realizamos este estudió para el que se utilizó un modelo descriptivo comparativo, en el que se evaluaron a 100 personas, de entre 15 a 18 años de edad con el Paquete de Valoración de la Creatividad (CAP) de Frank Williams y la Escala de Estilos de Socialización Parental en la Adolescencia (ESPA29). Los datos recolectados se procesaron con el programa estadístico SPSS, utilizando estadística descriptiva y estadística inferencial para comparar medias y varianzas, con el estadístico paramétrico ANOVA. Luego de comparar los puntajes obtenidos según el estilo parental en las variables pensamiento divergente y sentimiento divergente, se concluyó que no existen diferencias significativas en los niveles de pensamiento divergente según los estilos parentales de crianza, sin embargo, se encontró relación significativa entre los estilos parentales de crianza y el sentimiento divergente.

Palabras clave: Estilos parentales de crianza, pensamiento divergente, sentimiento divergente, creatividad.
\end{abstract}

\section{Abstract}

This article analyzed the association between parental styles and the divergent thinking and divergent feeling of teenagers. The family is an important influence in children's development and previous studies suggest that parental style can influence creativity. For this purpose, we have used a comparative descriptive 
model, in which we evaluated 10o people, between the ages of 15-18 years, with the Creativity Assessment Packet (CAP), authored by Frank Williams, and the Scale of Parental Socialization Styles in Teenagers (ESPA29). The collected data were processed with the SPSS software using descriptive and inferential statistics, to compare means and variances, with the parametric statistical ANOVA. After contrasting the different scores obtained in divergent thinking and divergent feeling according to parental styles, we concluded that there are not significant differences in the levels of divergent thinking by parenting style, but there is a significant association between parental styles and divergent feeling.

Keywords: parental styles, divergent thinking, divergent feeling and creativity.

\section{Introducción}

Siendo la familia la principal fuente de influencia para el desarrollo general de la persona humana es de importancia dedicar un espacio investigativo a temas que promuevan estilos educativos favorables para los hijos. Según Palet (2000) el núcleo familiar es donde se van adquiriendo los primeros hábitos, las habilidades y costumbres provistas de intensidad afectiva, que son conductas que nos acompañan a lo largo de nuestra vida, por ello los padres tienen un papel importante en la formación y desarrollo de los hijos.

Así mismo, es importante investigar las variables que puedan contribuir con el desarrollo del pensamiento y sentimiento divergentes, competencias útiles para el progreso de nuestra sociedad. En ese sentido, la presente investigación plantea la influencia de los estilos parentales de crianza sobre el desarrollo del pensamiento divergente y sentimiento divergente de adolescentes, realizándose con la medición de ambas variables para finalmente relacionarlas y comparar dentro de qué estilo parental de crianza se encuentran los mayores índices de desarrollo del pensamiento divergente y sentimiento divergente, que al ser los dos componentes de la creatividad, se han investigado relacionándolos a esferas cognitivas.

Analizando estudios que correlacionan inteligencia y creatividad, se sostiene que ambas variables se sirven de los mismos procesos cognitivos, pero se distinguen en los resultados, dado que el producto creativo está provisto de originalidad. Esto ha situado a la creatividad no solo en posesión de quienes se consideran genios o personas especiales, sino que, al implicar los mismos procesos cognitivos, es común a todos (Langley, 1987; Perkins, 1981; Weisberg, 1993).

Otras investigaciones similares han arrojado un bajo índice de correlación entre CI (Coeficiente Intelectual) y creatividad. Gatzels y Jackson, (1962) al investigar grupos de niños con un alto CI y altos puntajes en pensamiento divergente, encontraron que no habían diferencias en los resultados de las pruebas de logro, llegando a la conclusión de que la inteligencia y la creatividad son competencias aisladas, concepto que ayudó a la elaboración de tests que midieran la creatividad 
y contribuyeran a metodologías para el incremento de la misma. Se desarrolló así, la "teoría del Umbral" de Torrance (1962, 1963), que sostiene que existe un umbral de inteligencia mínimo para manifestarse creativo, un CI promedio. Para corroborar esta teoría se han efectuado diversas investigaciones, una de estas en la ciudad de Arequipa elaborada por Lewis y Peralta (2012), que explica la relación entre creatividad e inteligencia con estudiantes superdotados y de CI promedio. La muestra estuvo conformada por 34 estudiantes de ambos sexos entre 9 y 10 años, empleándose para la medición de las variables el Paquete de Valoración de la Creatividad (CAP) y el Test de Comprensión de Inteligencia no Verbal. Los resultados indicaron que no existe relación significativa entre inteligencia y creatividad, por tanto, un alto CI no es necesario para la manifestación de la creatividad.

Habiendo encontrado numerosos argumentos para la distinción de creatividad e inteligencia, se empezó a plantear el constructo de pensamiento divergente, siendo estudiado por diversos autores, como DeBono (1975) y Osborn (1972), quienes rechazaron la existencia de un único modo de resolución de problemas y plantearon el pensamiento divergente a partir de estudios comparativos que buscaban las diferencias entre éste y el pensamiento convergente. Dentro de las principales diferencias encontradas, el pensamiento divergente permite mayor flexibilidad en la búsqueda de solución a un problema mientras que el pensamiento convergente considera una única dirección que vislumbra como una solución clara desde el principio. Sin embargo, aunque habiendo estudiado las diferencias entre ambos tipos de pensamiento DeBono (1975) sostiene que «no se traba de decidir cuál es el más eficaz, ya que ambos son necesarios y se complementan mutuamente. Lo que importa es una perfecta conciencia de sus diferencias para facilitar la aplicación de ambos» (p. 54), lo que impulsó el estudio para encontrar elementos que pudieran distinguir las diferencias entre inteligencia y creatividad.

Consecuentemente, Guilford (1971, 1980) propuso cuatro factores para medir el pensamiento divergente. Estos elementos son: la originalidad o número de veces que una solución ha sido replicada en un contexto, la fluidez como el número de alternativas de resolución de un problema, la flexibilidad o capacidad para cambiar de perspectiva y la elaboración o grado de desarrollo de la idea producida. Dentro de estas cuatro dimensiones cognitivas de la creatividad se requiere de atención y memoria de información, ya que ambas intervienen dentro de todo el proceso creativo.

Se cree que las mentes creativas pueden abarcar mejor la información disponible para poder hacer resignificaciones, cambiar de categorías, pasar rápidamente de una idea a otra, para generar un producto novedoso. En función de esta concepción, se define el pensamiento divergente como el componente cognitivo de la creatividad, un medio para crear nuevas posibilidades, que nos permite retomar ideas pasadas y generar 
conexiones entre cuestiones aparentemente ajenas (Guilford, 1980; Mednick, 1962; Ruiz, 2004; Williams, 1980).

A partir de las ideas de Guilford (1971) se generan instrumentos de medición del pensamiento divergente desde la observación de los factores de fluidez, flexibilidad, originalidad y elaboración, antes mencionados. Sin embargo, aún se creía que la creatividad era la convergencia de factores cognitivos, de personalidad y la influencia del ambiente (Binet, 1986; Cattel, 1971; Gardner, 1983; Csikszentimihalyi, 1997). Es así que Williams (1980) desarrolla una teoría acerca de la creatividad que combina pensamiento divergentey agrega elementos de la esfera afectiva, los que engloba en el concepto de sentimiento divergente, que se podría definir como el componente afectivo de la creatividad, que nace de la convergencia de cuatro factores: la curiosidad, la imaginación, complejidad y la toma de riesgos (Williams, 1980).

Esto contribuyó a la idea del empleo de metodologías para el desarrollo o incremento de habilidades necesarias para el pensamiento divergente mediante técnicas que favorecen la flexibilidad mental ya su vez nos hace pensar en que el ambiente apropiado puede generar personas creativas (Gordon, 1992), motivo por el cual hemos decidido relacionar estas variables con los estilos parentales de crianza.

Dichos estilos son «esquemas prácticos que reducen las múltiples y minuciosas pautas educativas paternas a unas pocas dimensiones básicas que, cruzadas entre sí en diferentes combinaciones, dan lugar a diversos tipos habituales de educación familiar» (Baumrind, 1971, p. 88). Estas pautas de educación pretenden influir, modular y encausar la conducta de los hijos. En otras palabras, son una representación de la interacción habitual entre padres e hijos dentro del hogar y está conformada por la suma del estilo paterno y el estilo materno de crianza, y a su vez, buscan el control del impulso de los hijos, desarrollar su conciencia, prepararlos para le ejecución de roles sociales, transmitir valores o cultivar fuentes de significado (Wrong, 1994). También involucran socialización no formal; es decir, el estilo propio de vivir de la familia, sus valores y sus expectativas (Musitu \& García, 2004).

A partir de 1930 se van generando una diversidad de modelos teóricos, y trabajos sobre la socialización entre padres e hijos, sin embargo, los investigadores van encontrando una línea de pensamiento en común, con énfasis teórico en resolver la cuestión de si los padres recurrían al control o apego para socializar a sus hijos; generando modelos teóricos sobre la socialización parental que se explican a través de dos dimensiones que podrían resumirse en aceptación y control parental o factores aceptación/implicación y coerción/imposición (Baldwin, 1955; Becker 1964; MacCoby \& Levin, 1957; Schaefer, 1959; Symonds, 1939). El cruce de diferentes grados de estas dos dimensiones da lugar a la identificación de cuatro estilos paternos: el estilo autoritativo caracterizado por un equilibrio entre el alto autocontrol y alta afectividad, control firme y comunicación abierta con los hijos; el estilo autoritario, caracterizado por 
la alta coerción e imposición y falta de atención a las necesidades de los hijos; estilo negligente, caracterizado por una baja coerción y afecto, así como ausencia de límites; y por último, el estilo indulgente de padres comunicativos pero nada impositivos con las normas, que evitan la coerción e imposición. (Baumrind, 1971; Musitu \& García, 2004). Dentro de los tipos de socialización parental pueden presentarse inconsistencias entre los estilos de crianza paternos y maternos. Por lo general, esto sucede en familias en las que uno de los padres es más dominante, y se cree que esto puede influir de forma negativa en la transición de la familia a la independencia y al establecimiento de amistades. Dicha inconsistencia parental tiene a su vez relaciones significativas con alteraciones emocionales y conductuales como son mayores índices de sintomatología depresiva y agresión, tanto física como verbal y falta de control (Rodríguez et al., 2009; Vergara, 2002).

Así mismo, se han investigado las implicancias de los distintos estilos parentales de crianza sobre la parte emocional afectiva de los hijos saliendo más favorecidos aquellos criados mediante socialización autoritativa, desarrollando mayor confianza en sí mismos e internalizando mejor las normas sociales; mientras que, el estilo indulgente sin muchos límites, desarrolla en los hijos mayores problemas de conducta en la adolescencia (Jorge \& Gonzales, 2017). Según Llinares (1998), los hijos de padres indulgentes se muestran más inseguros y temerosos, característica que comparten con los hijos de padres autoritarios, quienes muestran resentimiento hacia sus padres, obediencia de las normas impregnada de miedo que no permite una buena internalización de las mismas. Por otro lado, los hijos de padres negligentes muestran consecuencias emocionales "invisibles" como el miedo al abandono, falta de confianza en los demás, pensamientos suicidas, baja autoestima, miedos irracionales, ansiedad y pobres habilidades sociales.

Otras investigaciones realizadas señalan que el estilo autoritativo se asocia con altos niveles de autoconcepto académico y autoconcepto emocional, que involucra una mejor percepción del aspecto físico propio y las capacidades físicas (Musitu \& García, 1994). También se ha encontrado que el significado del hijo dentro de la familia tanto como la percepción del sistema familiar se ve afectado negativamente, cuando se emplea el castigo físico, generando desajuste emocional y psicológico en el hijo (Bourque \& Elodi, 2006).

Retomando la idea inicial de la investigación, consideramos que ciertos estilos parentales de crianza pueden resultar de importancia vital para el desarrollo de la creatividad mientras que otros pueden ir en detrimento de ésta. Es por ello, que es necesario llevar a cabo mayores investigaciones que permitan la difusión de información para la adopción de estilos parentales más favorables.

Si bien es cierto, los factores de sentimiento y pensamiento divergente son difíciles de abordar debido a la cantidad de variables de tipo cognitivo, afectivo y 
ambientales que influyen en el desarrollo de las mismas; a su vez, parecieran haber consideraciones de valor para relacionarlas con los estilos parentales de crianza, ya que las investigaciones demuestran que el contexto juega un papel importante en el desarrollo creativo del individuo.

Existen pocas investigaciones en cuanto a esta materia, sin embargo, se han comparado usualmente desdeámbito educativo los modelos de aprendizaje que podrían influenciar en el desarrollo creativo de los niños. Ruiz (2004) determinó que los alumnos más creativos poseen estilos de aprendizaje más provechosos para la vida universitaria. Además, el mismo autor sostiene que no se encuentran significativamente relacionadas con la creatividad las variables de sexo, edad, origen social, ni con el tamaño del núcleo familiar o el nivel socioeconómico.

Palomeque y Ruiz (2012), al estudiar la relación entre los estilos cognitivos y los estilos parentales de crianza de estudiantes de básica primaria en Colombia, concluyeron que no existe correlación positiva entre los estilos cognitivos de los estudiantes y los estilos parentales de crianza. Por otro lado, la aplicación de métodos para poder incrementar los niveles de pensamiento divergente en el ámbito educativo, también abordados en una investigación colombiana, arrojaron que el uso de metodologías bisociativas en el aula está relacionado con altos niveles de pensamiento divergente, ya que promueven la elaboración de asociaciones remotas entre ideas que no tienen aparente conexión entre sí, genera razonamientos rápidos y trabaja sobre el análisis de ideas (RodriguezMartinez, 2013).

En el ámbito de la psicología educativa, en la ciudad de Arequipa los estudios realizados en estilos de aprendizaje y estilos parentales de crianza, han demostrado que en la educación secundaria básica regular de instituciones estatales, los alumnos prefieren el empleo de un estilo de aprendizaje de tipo divergente. A su vez que existe relación entre la modalidad de aprendizaje Experiencia Activa (EA) y el pensamiento divergente, más no entre las modalidad de aprendizaje de experiencia concreta (EC), observación reflexiva $(\mathrm{OR})$, conceptualización abstracta (CA) y pensamiento divergente (Pinares \& Toledo, 2000).

En otros estudios realizados por Ekvall (1999) en torno a la cultura creativa y clima organizacional se encontró que las características de las organizaciones que promueven el desarrollo creativo en sus empleados son el apoyo, la puesta en valor de tener iniciativa y el plantear desafíos. Lo que coincide con los resultados encontrados en investigaciones que medían la interacción paterno filial y el desarrollo creativo. Las familias de los niños altamente creativos suelen tener características de unidad, son estrechas, valoran la libertad de expresión, ambos padres suelen tener muy buena relación con sus hijos y los aceptan sin condiciones, incentivando la autonomía e independencia (Weisberg \& Springer, 1961). En esta línea, las investigaciones de Eisenbergm, Zhou, Spinrad, Valiente, 
Faber y Liewet (2005) muestran que los padres afectuosos crían hijos más adaptados y con menor frustración y problemas de agresión.

Lamana y De la Peña (2018), sostienen que $\mathbf{2 7 . 2} \%$ del rendimiento académico es explicado por la creatividad y el afrontamiento improductivo, donde ambos influyen de manera similar en el rendimiento académico, es decir, cuanto menos afrontamiento improductivo y cuanta más creatividad, más rendimiento académico. Lo que nos impulsa a buscar mejores métodos para influir y desarrollar la creatividad, ya que podría incrementar las oportunidades de los alumnos.

Si bien es cierto, los componentes de la creatividad están influidos por múltiples factores intrínsecos, ya sea de personalidad o motivacionales, también existen variables sociales que influyen de distinta manera en el proceso creativo, para los que se requiere el trabajo exhaustivo y la implicación personal. Amabile (1996), ha identificado dos clases de motivadores extrínsecos, los sinérgicos que aportan información y los motivadores extrínsecos no sinérgicos que proveen al sujeto la sensación de vigilancia, evaluación y crítica, que muchas veces genera bloqueos en la creatividad. En consecuencia, se sostiene que la creatividad se expresa comúnmente en ambientes que proveen de una buena cantidad de recursos a los sujetos y apoyo durante la ejecución de tareas (Amabile, 1996; Bloom, 1985; Stermberg \& Lubart, 1991).
Según investigaciones latinoamericanas la aceptación de los padres se relaciona en forma positiva con la creatividad y la disciplina laxa en forma negativa (Guillón et al., 2013), es decir, que el estilo parental que promueve aceptación es el que más se acerca al desarrollo óptimo de la creatividad.

La información comentada pareciera indicar que existe una relación entre los estilos de crianza y el desarrollo de habilidad creativas, y que determinados estilos parentales y prácticas familiares tienen incidencia positiva en el desarrollo de estas habilidades y conductas desde la primera infancia, mientras que otros estilos parentales bloquean este desarrollo (Martínez, 2012; Ruiz, 2004).

En consecuencia, consideramos que el tema de la influencia de los estilos parentales de crianza sobre el desarrollo global de los hijos es de interés para la comunidad científica, tanto como para la sociedad en general, ya que podría devolverse a la familia mayor responsabilidad sobre el desarrollo general de los hijos, labor que está en la actualidad delegada en mayor parte o totalmente a los centros educativos.

\section{Metodología}

\section{Diseño de investigación}

El trabajo empleado para conocer si existe asociación entre el pensamiento y sentimiento divergente y los estilos parentales de crianza se enmarcan en un estudio de tipo asociativo (Ato et al., 2013). 


\section{Muestra}

Se empleó un muestreo intencional no probabilístico (Hernández et al., 2010) de 100 sujetos pertenecientes a familias constituidas por ambos padres presentes en el hogar. Todos ellos estudiantes de ambos sexos ( 48 de sexo masculino y 52 de sexo femenino), de 15 a 18 años de edad, $15 \%$ de 15 años, 37\% de personas de 16 años, $26 \%$ de 17 años y $22 \%$ de 18 años; que se encontraban cursando el cuarto y quinto de secundaria de educación básica regular, y primer año de universidad, pertenecientes a instituciones tanto públicas como privadas.

\section{Instrumentos}

Para el recojo de información se utilizó el Paquete de Valoración de la Creatividad (CAP) creado por Frank Williams en 1980, traducido y adaptado por Portilla et al. (1993). Este instrumento consiste en dos test de administración grupal: una prueba de pensamiento divergente (formas A y B) $y$ una de sentimiento divergente.

El primer test produce puntajes para los cuatro factores de pensamiento divergente de fluidez, flexibilidad, originalidad y elaboración; derivados del factor analítico de Guilford (1971). Posee 12 trazos de alguna parte del dibujo para completar, así se les da a los sujetos numerosas posibilidades de asociación libre y expresión divergente, del mismo modo el hecho de indicar títulos inteligentes a los dibujos mide la habilidad verbal. En las pruebas de pensamiento divergente (forma A y B) se mide el tiempo para las producciones, que será 25 minutos para grados inferiores y 20 minutos para los superiores. Este test supone una forma completa de ver las habilidades de los sujetos en cuanto al pensamiento divergente (Williams, 1980).

El segundo test, es un cuestionario para la medición del sentimiento divergente. Deadministración individual ogrupal sin tiempo límite. Consiste en 50 preguntas a ser respondidas por el sujeto mismo, acerca de lo que considera como verdadero, parcialmente verdadero, menos verdaderoy aquello sobre lo que realmente no se puede decidir, con respecto de sí mismo. Las pruebas cuentan con una consistencia interna aceptable de $\alpha=.71$ para el test de pensamiento divergente y de $\alpha=.76$ para el test de sentimiento divergente (Lewis \& Peralta, 2012).

Por otro lado, se empleó la Escala de Estilos de Socialización Parental en la Adolescencia elaborada por Misitu y García (2004) en Madrid (España). Este es un cuestionario de 29 preguntas para marcar dos respuestas por pregunta, una referida a la conducta la madre y otra referida a la conducta del padre, en una escala de Likert que va desde "Siempre" (4) a "Nunca" (1). A partir de las puntuaciones se obtienen dos grandes dimensiones de aceptación/implicación y coerción/ imposición, en estas dos dimensiones se tipifica el estilo de socialización de cada padre (Estilo Paterno y Estilo Materno) como autoritario, autoritativo, indulgente o negligente, y si los padres tienen estilos distintos de criar al hijo se tiene un puntaje para la escala ambivalente. Cuenta con un índice de consistencia interna $\alpha=.968$ 
(Musitu \& García, 2004); y a su vez, en una investigación elaborada por Jarrín (2012) se procedió a realizar la validación de la misma a través de validez de contenido, utilizando para ello el método de juicio de expertos en familia de la Universidad Nacional Mayor de San Marcos, determinando que la escala pertenece a un dominio definido, así como también estos reactivos, que al ser tomados en conjunto tienen una proporción adecuada.

\section{Procedimientos}

Para el desarrollo de la presente investigación fue necesario realizar coordinaciones con distintas instituciones educativas de Arequipa que facilitaron la obtención de la muestra necesaria para la investigación. Es así que se evaluó a 250 adolescentes de entre 15 a 18 años de edad, de los cuales solo 100 llenaron adecuadamente los instrumentos y pudieron ser incluidos en la muestra. Para la recolección de datos se ingresó a cada una de las clases en el horario escolar, pidiendo la colaboración anónima y voluntaria de los participantes, a quienes se les hizo firmar un consentimiento informado, mientras que para el caso de los menores de edad, fueron los padres quienes dieron su conformidad.

\section{Análisis de datos}

Los test aplicados, Escala de valoración del pensamiento divergente de Williams (CAP) - Forma A y Cuestionario de Sentimiento Divergente, y Escala de Estilos de Socialización Parental en la Adolescencia, fueron calificados por medio de parrillas de corrección electrónicas, especialmente elaboradas para la presente investigación. La base de datos fue construida y procesada con el programa estadístico SPSS versión 21, mediante la aplicación de pruebas de estadística inferencial.

\section{Resultados}

Como observamos en la Tabla 1, según la media y la desviación estándar de los estilos parentales de crianza, los hijos de padres con estilo de crianza negligente $(\mathrm{M}=76,17 ; \mathrm{DE}=18,029)$ han obtenido los puntajes más bajos, sin embargo, no se evidencian grandes diferencias según los estilos parentales de crianza para el pensamiento divergente. 
Tabla 1. Índices de pensamiento y sentimiento divergente según estilo parental

\begin{tabular}{|lllccc|}
\hline & & Media & $\begin{array}{c}\text { Desviación } \\
\text { estándar }\end{array}$ & $\begin{array}{c}\text { 95\% del intervalo de } \\
\text { confianza para la media } \\
\text { Límite } \\
\text { inferior }\end{array}$ & $\begin{array}{c}\text { Límite } \\
\text { superior }\end{array}$ \\
\hline & Autoritario & 78,50 & 12,598 & 65,28 & 91,72 \\
& Autoritativo & 76,75 & 12,703 & 66,13 & 87,37 \\
Pensamiento & Negligente & 76,17 & 18,029 & 64,71 & 87,62 \\
& Indulgente & 78,31 & 12,539 & 74,70 & 81,91 \\
& Ambivalente & 76,80 & 11,303 & 72,13 & 81,47 \\
& Total & 77,56 & 12,787 & 75,02 & 80,10 \\
\hline \multirow{2}{*}{$\begin{array}{l}\text { Sentimiento } \\
\text { divergente }\end{array}$} & Autoritario & 74,33 & 8,756 & 65,14 & 83,52 \\
& Autoritativo & 74,75 & 8,714 & 67,47 & 82,03 \\
& Negligente & 62,08 & 9,885 & 55,80 & 68,36 \\
& Indulgente & 70,16 & 8,547 & 67,71 & 72,62 \\
& Ambivalente & 71,20 & 7,821 & 67,97 & 74,43 \\
& Total & 70,07 & 9,015 & 68,28 & 71,86 \\
\hline
\end{tabular}

Por otro lado, en la parte inferior de la Tablaı, se observa las medias y la desviación estándar según sentimiento divergente, en la cual el estilo negligente $(\mathrm{M}=62,08 ; \mathrm{DE}=9,885)$ sigue obteniendo la media más baja, mientras que la media más alta es la del estilo autoritativo $(\mathrm{M}=$ $74,75 ; \mathrm{DE}=8,714)$. Además, se muestra que hay mayor diferencia entre las medias obtenidas por los distintos grupos para sentimiento divergente. Es decir, el estilo autorizativo está positivamente relacionado con el desarrollo del sentimiento divergente mientras que el estilo negligente se encuentra en el pico más bajo del desarrollo de sentimiento divergente.
Los resultados del ANOVA nos muestran un valor $\mathrm{F}$ de o,111 y una significancia de $\mathrm{p}=.978$ para pensamiento divergente, lo que nos confirma que no hay diferencias significativas. Esto nos permite decir que parece no existir influencia de los estilos parentales de crianza sobre el pensamiento divergente de los hijos. En lo concerniente al sentimiento divergente según los estilos parentales de crianza, los resultados del ANOVA nos dan un valor a $\mathrm{F}$ de 3,690 y una significancia de $\mathrm{p}=.008$. Es decir que las diferencias observadas entre grupos son estadísticamente significativas, permitiéndonos concluir que los estilos de crianza sí influyen sobre el sentimiento divergente de los hijos. 
Tabla 2. ANOVA del pensamiento divergente y sentimiento divergente según estilos parentales

\begin{tabular}{|llccccc|}
\hline & & $\begin{array}{c}\text { Sumade } \\
\text { cuadrados }\end{array}$ & gl & $\begin{array}{c}\text { Media } \\
\text { cuadrática }\end{array}$ & F & Sig. \\
\hline Pensamiento & Entre grupos & 75,565 & 4 & 18,891 & 0,111 & .978 \\
divergente & Dentro de grupos & 16111,075 & 95 & 169,590 & & \\
& Total & 16186,640 & 99 & & & \\
\hline \multirow{2}{*}{$\begin{array}{l}\text { Sentimiento } \\
\text { divergente }\end{array}$} & Entre grupos & 1082,066 & 4 & 270,517 & 3,690 & .008 \\
& Dentro de grupos & 6964,444 & 95 & 73,310 & & \\
\hline
\end{tabular}

Si bien no se encontró una relación esta- autoritario y la más alta 83,40 del autoridísticamente significativa, se observa tativo. Demostrando así, la importancia cierta diferencia entre las medias obte- del padre sobre la dimensión cognitiva nidas porvarones en pensamiento diver- de la creatividad en los hijos varones gente, siendo la menor 69,0o del estilo (ver Tabla 3).

Tabla 3. Medias de pensamiento divergente y sentimiento divergente según estilo paterno en varones

\begin{tabular}{|c|c|c|c|c|c|}
\hline & & \multirow{2}{*}{ Media } & \multirow{2}{*}{$\begin{array}{l}\text { Desviación } \\
\text { estándar }\end{array}$} & \multicolumn{2}{|c|}{$\begin{array}{c}95 \% \text { del intervalo } \\
\text { de confianza para la media }\end{array}$} \\
\hline & & & & $\begin{array}{l}\text { Límite } \\
\text { inferior }\end{array}$ & $\begin{array}{l}\text { Límite } \\
\text { superior }\end{array}$ \\
\hline \multirow{5}{*}{$\begin{array}{l}\text { Pensamiento } \\
\text { divergente }\end{array}$} & Autoritario & 69,00 & 4,243 & 30,88 & 107,12 \\
\hline & Autoritativo & 83,40 & 3,847 & 78,62 & 88,18 \\
\hline & Negligente & 78,73 & 12,362 & 70,42 & 87,03 \\
\hline & Indulgente & 77,37 & 11,500 & 73,07 & 81,66 \\
\hline & Total & 77,96 & 11,076 & 74,74 & 81,17 \\
\hline \multirow{5}{*}{$\begin{array}{l}\text { Sentimiento } \\
\text { divergente }\end{array}$} & Autoritario & 73,50 &, 707 & 67,15 & 79,85 \\
\hline & Autoritativo & 72,60 & 10,738 & 59,27 & 85,93 \\
\hline & Negligente & 69,27 & $6,35^{8}$ & 65,00 & 73,54 \\
\hline & Indulgente & 68,47 & 7,912 & 65,51 & 71,42 \\
\hline & Total & 69,29 & 7,707 & 67,05 & 71,53 \\
\hline
\end{tabular}

Los resultados del ANOVA dan una significancia de .460 en pensamiento divergente y .615 en sentimiento divergente. Es decir que las diferencias observadas al comparar las medias no son estadísticamente significativas. 
Tabla 4. ANOVA de pensamiento divergente y sentimiento divergente según estilo paterno en varones

\begin{tabular}{|llccccc|}
\hline & & $\begin{array}{c}\text { Suma } \\
\text { de cuadrados }\end{array}$ & gl & $\begin{array}{c}\text { Media } \\
\text { cuadrática }\end{array}$ & F & Sig. \\
\hline \multirow{2}{*}{$\begin{array}{l}\text { Pensamiento } \\
\text { divergente }\end{array}$} & Entre grupos & 325,568 & 3 & 108,523 & 0,878 & .460 \\
& Dentro de grupos & 5440,348 & 44 & 123,644 & & \\
\hline \multirow{2}{*}{$\begin{array}{l}\text { Sentimiento } \\
\text { divergente }\end{array}$} & Entre grupos & 5765,917 & 47 & & & \\
& Dentro de grupos & 2681,348 & 44 & 60,940 & & \\
& Total & 2791,917 & 47 & & & \\
\hline
\end{tabular}

En la Tabla 5 se muestra el análisis descrip- análisis descriptivo de sentimiento divertivo del pensamiento divergente por grupo gente por grupo según el estilo materno, según el estilo materno, siendo la media mostrando los puntajes más bajos para más baja la del estilo negligente $(M=$ los estilos negligente $(M=69,50)$ e indul$73,83)$, no evidenciándose grandes dife- gente $(M=68,64)$; y al igual que con el rencias entre los grupos. Así mismo, en pensamiento divergente no se evidencian la parte inferior de cuadro se muestra el grandes diferencias entre grupos.

Tabla 5. Medias en pensamiento y sentimiento divergente según estilo materno en varones

\begin{tabular}{|c|c|c|c|c|c|}
\hline & & \multirow{2}{*}{ Media } & \multirow{2}{*}{$\begin{array}{l}\text { Desviación } \\
\text { estándar }\end{array}$} & \multicolumn{2}{|c|}{$\begin{array}{c}95 \% \text { del intervalo } \\
\text { de confianza para la media }\end{array}$} \\
\hline & & & & $\begin{array}{l}\text { Límite } \\
\text { inferior }\end{array}$ & $\begin{array}{l}\text { Límite } \\
\text { superior }\end{array}$ \\
\hline \multirow{5}{*}{$\begin{array}{l}\text { Pensamiento } \\
\text { divergente }\end{array}$} & Autoritario & 86,00 & 0.000 & 0.000 & 0.000 \\
\hline & Autoritativo & 79,63 & 6,346 & 74,32 & 84,93 \\
\hline & Negligente & 73,83 & 8,704 & 64,70 & 82,97 \\
\hline & Indulgente & 78,06 & 12,394 & 73,67 & 82,46 \\
\hline & Total & 77,96 & 11,076 & 74,74 & 81,17 \\
\hline \multirow{5}{*}{$\begin{array}{l}\text { Sentimiento di- } \\
\text { vergente }\end{array}$} & Autoritario & $75, \mathrm{oo}$ & 0.000 & 0.000 & 0.000 \\
\hline & Autoritativo & 71,13 & 8,935 & 63,65 & 78,60 \\
\hline & Negligente & 69,50 & 4,764 & 64,50 & 74,50 \\
\hline & Indulgente & 68,64 & 7,995 & 65,80 & 71,47 \\
\hline & Total & 69,29 & 7,707 & 67,05 & 71,53 \\
\hline
\end{tabular}


En la Tabla 6 la prueba de ANOVA nos muestra una significancia de .686 para pensamiento divergentey una significancia de .755 para sentimiento divergente. Es decir que no se encuentra ninguna asociación entre los componentes cognitivo ni afectivo de la creatividad del hijo varón y el estilo de crianza materno.

Tabla 6. ANOVA de pensamiento divergente según estilo materno en varones

\begin{tabular}{|llccccc|}
\hline & & $\begin{array}{c}\text { Suma } \\
\text { de cuadrados }\end{array}$ & gl & $\begin{array}{c}\text { Media } \\
\text { cuadrática }\end{array}$ & F & Sig. \\
\hline Pensamiento & Entre grupos & 189,330 & 3 & 63,110 & 0,498 & .686 \\
divergente & Dentro de grupos & 5576,587 & 44 & 126,741 & & \\
& Total & 5765,917 & 47 & & & \\
\hline \multirow{2}{*}{$\begin{array}{l}\text { Sentimiento } \\
\text { divergente }\end{array}$} & Entre grupos & 73,905 & 3 & 24,635 & 0,399 & .755 \\
& Dentro de grupos & 2718,011 & 44 & 61,773 & & \\
\hline
\end{tabular}

Como observamos en la Tabla 7, las medias en pensamiento divergente de las hijas mujeres según el estilo paterno son similares. En cuanto a las medias obtenidas en sentimiento divergente encontramos mayores puntajes si provienen de un hogar con estilos autoritativo ( $M=74.20)$ o autoritario $(M=74,56)$, mientras que los menores puntajes se encuentran en el estilo negligente $(M=64,00)$ e indulgente $(M=72,28)$.

Tabla 7. Medias en pensamiento y sentimiento divergente según estilo paterno en mujeres

\begin{tabular}{|lllccc|}
\hline & & Media & $\begin{array}{c}\text { 95\% del intervalo } \\
\text { estándar }\end{array}$ & $\begin{array}{c}\text { 95 confianza para la media } \\
\text { Límite } \\
\text { inferior }\end{array}$ & $\begin{array}{c}\text { Límite } \\
\text { superior }\end{array}$ \\
\hline \multirow{2}{*}{$\begin{array}{lllcl}\text { Pensamiento } \\
\text { divergente }\end{array}$} & Autoritario & 79,33 & 10,920 & 70,94 & 87,73 \\
& Autoritativo & 68,00 & 17,903 & 45,77 & 90,23 \\
& Indulgente & 77,56 & 13,251 & 72,09 & 88,81 \\
& Total & 77,19 & 14,284 & 73,22 & 81,03 \\
\hline \multirow{2}{*}{$\begin{array}{l}\text { Sentimiento } \\
\text { divergente }\end{array}$} & Autoritario & 74,56 & 8,428 & 68,08 & 81,03 \\
& Autoritativo & 74,20 & 6,834 & 65,71 & 82,69 \\
& Negligente & 64,00 & 12,152 & 56,66 & 71,34 \\
& Indulgente & 72,28 & 8,735 & 68,67 & 75,89 \\
\hline
\end{tabular}


Los resultados del ANOVA para comparar las medias obtenidas por mujeres en pensamiento divergente según estilo paterno nos dan una significancia de .504, es decir que las diferencias halladas en las medias de pensamiento divergentes según estilo paterno, no son estadísticamente significativas. Sin embargo, podemos afirmar que el sentimiento divergente de la hija mujer sí se encuentra influido por el estilo paterno, ya que el valor de la significancia es de .036, lo que evidencia una relación estadísticamente significativa entre la variable sentimiento divergente de las hijas mujeres y el estilo paterno (ver Tabla 8).

Tabla 8. ANOVA en pensamiento y sentimiento divergentes según estilo paterno en mujeres

\begin{tabular}{|llccccc|}
\hline & & $\begin{array}{c}\text { Suma } \\
\text { de cuadrados }\end{array}$ & gl & $\begin{array}{c}\text { Media } \\
\text { cuadrática }\end{array}$ & F & Sig. \\
\hline Pensamiento & Entre grupos & 490,686 & 3 & 163,562 & 0,792 & .504 \\
divergente & Dentro de grupos & 9915,391 & 48 & 206,571 & & \\
& Total & 10406,077 & 51 & & & \\
\hline \multirow{2}{*}{$\begin{array}{l}\text { Sentimiento } \\
\text { divergente }\end{array}$} & Entre grupos & 840,611 & 3 & 280,204 & 3,086 & .036 \\
& Dentro de grupos & 4358,062 & 48 & 90,793 & & \\
\hline
\end{tabular}

En la Tabla 9 se muestra el análisis descriptivo del pensamiento divergente por grupos según el estilo materno hacia la hija mujer no evidenciándose grandes diferencias en las medias de pensamiento divergente según el estilo del padre hacia la hija. Sin embargo, el análisis de las medias de la variable sentimiento divergente según el estilo de la madre obtenido por las mujeres muestra que el estilo autoritativo $(M=78,43)$ de la madre está asociado con los mayores puntajes en sentimiento divergente de la hija mujer, mientras que las hijas criadas en estilos indulgente $(M=72,18)$ y negligente $(M=62,91)$ han obtenido puntajes más bajos. Evidenciándose una alta fluctuación de los puntajes obtenidos en función al estilo de crianza materno, siendo el estilo materno autoritativo el de influencia más positiva para el desarrollo del sentimiento divergente de las hijas mujeres y el estilo negligente el menos productivo. 
Tabla 9. Medias obtenidas en pensamiento divergente según estilo materno en las mujeres

\begin{tabular}{|c|c|c|c|c|c|}
\hline & & Media & Desviación & $\begin{array}{r}95 \% \text { del int } \\
\text { pa }\end{array}$ & $\begin{array}{l}\text { e confianza } \\
\text { dia }\end{array}$ \\
\hline & & vecua & estándar & $\begin{array}{l}\text { Límite } \\
\text { inferior }\end{array}$ & $\begin{array}{c}\text { Límite } \\
\text { superior }\end{array}$ \\
\hline & Autoritario & 77,17 & 11,788 & 64,80 & 89,54 \\
\hline & Autoritativo & 74,71 & 14,326 & 61,46 & 87,96 \\
\hline $\begin{array}{l}\text { Pensamiento } \\
\text { divergente }\end{array}$ & Negligente & 74,09 & 18,705 & 61,52 & 86,66 \\
\hline & Indulgente & 79,04 & 13,245 & 73,90 & 84,17 \\
\hline & Total & 77,19 & 14,284 & 73,22 & 81,17 \\
\hline & Autoritario & 69,83 & 11,232 & 58,05 & 81,62 \\
\hline & Autoritativo & 78,43 & 3,735 & 74,97 & 81,88 \\
\hline $\begin{array}{l}\text { Sentimiento } \\
\text { divergente }\end{array}$ & Negligente & 62,91 & 12,037 & 54,82 & 71,00 \\
\hline & Indulgente & 72,18 & 8,354 & 68,94 & 75,42 \\
\hline & Total & 70,79 & 10,096 & 67,98 & 73,60 \\
\hline
\end{tabular}

La prueba ANOVA para comparar el pensamiento divergente según el estilo materno en las mujeres nos da un valor F de 0,384 cuya significancia es de .765 , es decir que no existeasociación estadísticamente significativa entreambas variables. El estilo materno entonces, no influye sobre el pensamiento divergente de las hijas mujeres. Por otro lado, al comparar sentimiento divergente según estilo materno en mujeres nos da un valor F de 4,550 cuya significancia es de .oo7, mostrando una asociación estadísticamente significativa entre ambas variables, por lo que podríamos decir que el estilo materno influye en el sentimiento divergente (ver Tabla 10).

Tabla 1o. ANOVA de pensamiento y sentimiento divergente según estilo materno en mujeres

\begin{tabular}{|llccccc|}
\hline & & $\begin{array}{c}\text { Suma de } \\
\text { cuadrados }\end{array}$ & gl & $\begin{array}{c}\text { Media } \\
\text { cuadrática }\end{array}$ & F & Sig. \\
\hline Pensamiento & Entre grupos & 243,942 & 3 & 81,314 & 0,384 & .765 \\
divergente & Dentro de grupos & 10162,135 & 48 & 211,711 & & \\
& Total & 10406,077 & 51 & & & \\
\hline Sentimiento & Entre grupos & 1151,109 & 3 & 383,703 & 4,550 & .007 \\
divergente & Dentro de grupos & 4047,564 & 48 & 84,324 & & \\
& Total & 5198,673 & 51 & & & \\
\hline
\end{tabular}




\section{Discusión}

En el presente estudio, tras comparar los niveles de pensamiento divergente y sentimiento divergente con los resultados obtenidos según los estilos parentales de crianza, se ha podido determinar que existen diferencias significativas en el sentimiento divergente según sea el estilo de crianza de los padres. Sin embargo, no existe asociación estadísticamente significativa entre el pensamiento divergentey los estilos parentales de crianza, por lo que parece ser que la influencia del entorno familiar se centra en la dimensión afectiva de los hijos (Palet, 2000).

Como vemos en el caso del sentimiento divergente, cuyos indicadores son de tipo afectivo, la relación con los estilos parentales de crianza es mucho mayor que la encontrada con la variable pensamiento divergente. Creemos que esto se debe a que esta última posee factores de tipo cognitivo, cuyo desarrollo es responsabilidad en gran parte de las escuelas, principales actores en el desarrollo cognitivo y formación del pensamiento de sus alumnos.

Ahora bien, el rol de los colegios no anula la influencia de los padres sobre la dimensión cognitiva de la creatividad de los hijos, ya que existe cierta asociación entre el pensamiento divergente de los hijos varones y el estilo de crianza del padre. Además, si analizamos las medias obtenidas según los distintos estilos de crianza paternos, veremos una asociación positiva entre el estilo de crianza de tipo autoritativo y el pensamiento divergente de los hijos varones. Por el contrario, en el caso de los padres autoritarios, se ve que la asociación es negativa, ya que va en detrimento de los niveles de pensamiento divergente. Esto evidencia la importancia del rol paterno en el desarrollo cognitivo de los hijos, ya que si no se promueve el diálogo y la reflexión (estilo autoritativo) se puede generar un niño autómata que cumple normas estrictamente sin haber razonado sobre ellas (estilo autoritario).

Pasando al sentimiento divergente, la asociación significativa encontrada entre el factor afectivo de la creatividad y los estilos parentales de crianza, encuentra un sustento teórico en Mercedes Palet (2000), quien plantea que el niño es fruto del amor esponsal de sus padres, y desde que empieza a existir pasa a ser parte integrante de esta unión afectiva. Es así, como en su núcleo familiar experimenta los efectos de una convivencia familiar amorosa o de la ausencia de ésta. Es lógico entonces, que un modelo afectivo correcto promueva un afecto sano en el niño.

Si analizamos los puntajes obtenidos en sentimiento divergente por los sujetos con padres negligentes e indulgentes, encontramos que se trata de valores inferiores, lo que nos permite establecer que dichos estilos están en detrimento del factor afectivo de la creatividad. El estilo negligente es caracterizado por baja aceptación/implicación y baja coerción/ imposición, por lo que los padres de este estilo se muestran ausentes. En este caso el sentimiento divergente, altamente relacionado con curiosidad y toma de riesgo, se ve afectado dando lugar a la indecisión 
de los hijos, característica que se contrapone a la creatividad, esta incertidumbre se genera ya que los padres dejan al hijo en libertad para hacer lo que le plazca. El niño al no tener pautas ni reglas para distinguir lo bueno de lo malo, carece de certezas aun cuando se le cuestiona acerca de sí mismo, de sus gustos o preferencias.

Esto no sucede en el caso del estilo de crianza autoritario, en el cual, a pesar de ser un estilo negativo, se han hallado niveles altos de sentimiento divergente. Lo cual se explicaría por la alta exigencia y coerción de los padres que no deja lugar a la duda sobre lo que se tiene o se quiere hacer el niño. Del mismo modo, el estilo indulgente ha afectado de forma negativa los puntajes en sentimiento divergente de los hijos. Al igual que en caso de los padres negligentes, la baja coerción/imposición genera indecisión en los hijos. Pero, este no es el único problema, sino que la alta aceptación/implicación característica de este estilo produce además conformismo. Los padres indulgentes hacen sentir a los niños que todo lo que hacen está bien y que, aunque sea poco o mediocre es suficiente, por ende, no los animan a buscar nuevas cosas, ni a dar lo mejor de sí. Lo que se contrapone con ciertas dimensiones del sentimiento divergente, como son la curiosidad, la imaginación y la complejidad.

Por otro lado, hemos hallado asociaciones significativas entre el estilo de crianza materno y el sentimiento divergente en las hijas mujeres. Si nos detenemos en las medias obtenidas según los diferentes estilos maternos encontraremos que las hijas con madres autoritativas han obtenido los puntajes más altos. Creemos que esto puede deberse a que la familia es el lugar donde se adquiere la seguridad. El niño se descubre amado y valioso dentro de su seno familiar, se da cuenta que tiene algo que donar a los demás y que puede hacerlo libremente, porque su familia lo acoge con amor.

Los padres autoritativos, son justamente así, se involucran afectivamente con sus hijos, propician el diálogo sin perder la autoridad propia de su rol, es por ello que los niños criados por padres con este estilo, pueden explorar sin miedo, ser más imaginativos, indagar problemas intrincados, e incluso tomar ciertos riesgos. Ello se debe a que saben que sus padres estarán ahí para protegerlos, pero también para cuestionarlos y ponerles límites. Además, es importante destacar el valor del diálogo como forma esencial de comunicación en la familia, ya que a través de éste se da a conocer la implicancia y afecto de los padres, las necesidades e ideas de los hijos, y se favorece la adquisición de hábitos buenos (conscientes yvoluntarios) en vez de costumbres monótonas. Dados los argumentos anteriormente discutidos, sostenemos que un estilo parental equilibrado, que se manifiesta en una adecuada combinación entre los ejes aceptación/ implicación y coerción/imposición, es crucial en el desarrollo de la parte afectiva de la creatividad.

Finamente, debe tenerse presente que la muestra ha sido pequeña y por tanto, los resultados no pueden generalizarse. Por ello, se recomiendan 
futuras investigaciones con muestras más representativas que versen sobre la influencia de la madre en el sentimiento divergente de los hijos y la influencia del padre sobre el pensamiento divergente, y otras investigaciones que puedan aportar como sustento teórico para que las escuelas puedan reafirmar la labor que estás tienen como agente en la formación cognitiva de los niños y adolescentes; y al mismo tiempo, pedir la participación activa por parte de los padres de familia para el desarrollo óptimo de los niños.

\section{Financiamiento}

La presente investigación fue autofinanciada.

\section{Conflictos de interés}

La autora declara que no tiene conflictos de interés. 


\section{Referencias}

Amabile, T. (1996). Creatividad en contexto. Bloulder.

Ato, M., López, J., \& Benavente, A. (2013). Un sistema de clasificación de los diseños de investigación en psicología. Anales de Psicología, 29(3), 1038-1059. http://www. redalyc.org/articulo.oa?id=16728244043

Baumrind, D. (1971). Current patterns of parental authority. Devolopmental Pyschology, 4(2), 1-103. https://doi.org/10.1037/hoo30372

Bladwin, A. (1955). Comportamiento y desarrollo en la niñez. Dryden Press.

Binet, A., \& Henri, V. (1896). La psicología individual. Annee Psychoologique, 2.

Bloom, B. (1985) Desarrollando talento en gente joven. Ballantine.

Bourque, S., \& Elordi, C. (2006). Relations among corporal punishment, perceived parental acceptance, and psychological adjustment in Jamaican youths. CrossCultural Research, 40(3), 268-286. https://doi.org/10.1177/1069397105284397

Cattell, R. B. (1971). Abilities: Their structure, growth and action. Houghton-Mifflin.

Csikszentmihalyi, M. (1997). Creatividad. Harper Collins.

De Bono, E. (1975). Lateral thinking for management. McGraw-Hill.

Eisenberg, N., Zhou, Q., Spinrad, T. L., Valiente, C., Faber, R. A., \& Liew, J. (2005). Relations among positive parenting, children's effortful control, and externalizing problems: A three-wave longitudinal study. Child Development, 76(5), 1055-1071. https://doi.org/10.1111/j.1467-8624.2005.00897.x

Ekvall, G. (Ed.). (1999). Creative climate. Encyclopedia of Creativity. 1, 403-412.

Gardner, H. (1983). Frames of mind: the theory of multiple intelligences. Basic.

Getzels, J., \& Jackson, P. (1962) Creatividad e inteligencia. Exploración con alumnos superdotados. Wiley.

Guilford, J. P. (1971). The nature of human intelligence. McGraw-Hill. 
Guilford, J. P. (1980). La creatividad. Narcea.

Guillón, S., Vargas-Rubilar, J., \& Krumm, G. (2013). Estilos parentales y creatividad en niños escolarizados. Psicoperspectivas. Individuo y Sociedad, 12(1), 161-182. http:// dx.doi.org/10.5027/psicoperspectivas-Vol12-Issue1-fulltext-223

Gordon, W. J. (1992). Sinéctica: historia, evolución y métodos. Paidós.

Hernández, R., Fernández, C., \& Baptista, P. (2010). Metodología de la investigación. McGraw-Hill Interamericana.

Jarrín, O. (2012). Estilos de socialización padre hijo desde temprana edad y la comunicación de su orientación sexual al padre por adolescentes varones homosexuales. (Tesis para optar por el Título de Licenciado en Psicología). Universidad Nacional Mayor de San Marcos, Lima, Perú.

Jorge, E., \& Gonzales, C. (2017). Estilos de crianza parental: una revisión teórica. Informes Psicológicos, 17(2), 39-66.

Lamana, M., \& De la Peña, C. (2018). Rendimiento académico en matemáticas. Relación con creatividad y estilos de afrontamiento. Revista Mexicana de Investigación Educativa, 23(79), 1075-1092. https://www.redalyc.org/journal/140/14059462003/ html/

Langley, P. (1987). Scientific discovery: Computational explorations of the creative processes. MIT Press.

Lewis, P., \& Peralta, C. (2012). Creatividad en estudiantes superdotados y de inteligencia promedio. (Tesis para optar por el título de Licenciado en Psicología). Universidad Católica Santa María, Arequipa, Perú.

Llinares, L. (1998). La configuración del autoconcepto y los valores en el contexto familiar. (Tesis para optar por el grado de Doctor en Psicología). Universidad de Valencia, España.

Martínez, E. (2012), Interacción de la creatividad con los estilos de aprendizaje en estudiantes universitarios de pedagogía de Galicia. Revista Iberoamericana sobre Calidad, Eficacia y Cambio en Educación, 10(2), 180-200.

Maccoby, E. E., \& Martin, J. A. (1983). Socialization in the context of the family: Parent-child interaction. Wiley. 
Musitu, G., \& Garcia, F. (2004). ESPA 29 Escala de Estilos de Socialización Parental en la Adolescencia. TEA.

Mednick, S. A. (1962). The associates basis of the creative process. En Romo-Santos, M. Interpretación asociativa del proceso creador. UAM.

Musitu, G., García, F., \& Gutiérrez, M. (1994). AFA: Autoconcepto forma A [AFA: Self-concept form A]. TEA.

Osborn, A. (1953). Applied imagination. Scribner's Press.

Palet, M. (2000). Familia, educadora del ser humano. Balmes.

Palomeque, Y., \& Ruiz, G. (2012). Estilos cognitivos de estudiantes de básica primaria y su relación con los estilos parentales. Plumilla Educativa, 271-292.

Perkins, D. (1981). The mind's best work. Harvard University.

Pinares, J., \& Toledo, V. (200o). Relación entre los estilos de aprendizaje u el pensamiento divergente investigación realizada con los alumnos adolescentes varones del 4 to y 5 to año. (Tesis para optar por la Licenciatura en Psicología). Universidad Nacional San Agustín, Arequipa, Perú.

Portilla, C. et al. (1993). Paquete de valoración de la creatividad (CAP). Traducido y adaptado a la cultura latinoamericana. Universidad Católica Santa María.

Rodríguez, M., Del Barrio, M., \& Carrasco, M. (2009). ¿Cómo perciben los hijos la crianza materna y paterna? Escritos de Psicología, 2(2), 10-18. https://scielo.isciii. es/pdf/ep/v2n2/original2.pdf

Rodríguez-Martínez, G. (2013). Bisociaciones creativas, sinéctica y pensamiento divergente. Revista $Q, 8(15)$, 1-22. https://repository.upb.edu.co/bitstream/ handle/20.50o.11912/6816/Bisociaciones\%2ocreativas $\% 2 C \% 20 \sin \% \mathrm{C}_{3} \%$ Agctica\%20 y\%2opensamiento\%2odivergente.pdf?sequence $=1 \&$ isAllowed $=\mathrm{y}$

Ruiz, C. (2004). Creatividad y estilos de aprendizaje. (Tesis para optar por el grado de Doctor). Universidad de Málaga, España.

Schaefer, E. (1959). A circumflex model for maternal behavior. Journal of Abnormal and Social Psychology, 59, 226-235. 
Sternberg, R., \& Lubart, T (1991). An investment theory of creativity and its development. Human Development, 34, 1-32. https://www.jstor.org/stable/26767348

Symonds, P. (1939). La psicología de las relaciones parento-filiales. Appleton-Century-Croft.

Torrance, P. (1962). Guiding creativity talent. Prentice Hall.

Torrance, P. (1963). Exploración en pensamiento creativo, en los primeros años de escuela. Un informe sobre los progresos. Wiley.

Vergara, C. (2002). Creencias relacionadas con las prácticas de la crianza de los hijos/ as. Universidad de Colima.

Weisberg, P. (1993). Creatividad, más allá del mito de los genios. Freeman.

Weisberg, P., \& Springer, K. (1961). Environmental factors in creative function. Archives of General Psychiatry, 5(6), 554-564. https://doi.org/10.1001/ archpsyc.1961.01710180038005

Williams, F. (1980). Creativity assessment package. Shoal Creek Boulevard.

Wrong, D. (1994). El problema del orden: ¿Qué une y divide a la sociedad? Free Press.

Recibido: 29 de setiembre de 2020

Revisado: 24 de marzo de 2021

Aceptado: 10 de octubre de 2021 